\title{
ANTARCTIC ATMOSPHERIC TRANSPARENCY AT INFRARED AND MILLIMETRE WAVELENGTHS
}

\author{
John Bally \\ University of Colorado and Center for Astrophysical Research in Antarctica
}

The Antarctic Plateau is the best site on Earth for astrophysical research from millimetre to near-infrared wavelengths. The low temperature, high elevation on top of the ice sheet, and the Polar Vortex, which blocks the infusion of moist air, result in extremely low column densities of atmospheric water vapour. These unique conditions may result in the clearest and darkest sky available from the surface of the Earth in this wavelength range. Under clear-sky conditions, ground based observations in the 1 to $1000 \mu \mathrm{m}$ spectral range are hampered by absorption lines of water and the thermal emission from the atmosphere, telescope, and its environment. The precipitable water column above the South Pole (elevation $2800 \mathrm{~m} ;(\approx 3500 \mathrm{~m}$ pressure altitude) is almost always less than $1 \mathrm{~mm}$, averages about $0.3 \mathrm{~mm}$, and can sometimes be under $0.1 \mathrm{~mm}$, making possible observations in many sub-mm and IR windows. Even drier conditions are expected on the highest parts of the continent. A flat ice plateau near $-82^{\circ} \mathrm{S}$ latitude, $72^{\circ} \mathrm{E}$ longitude contains thousands of square miles at an altitude above $4000 \mathrm{~m}(\approx 5000 \mathrm{~m}$ pressure altitude $)$, and is a possible site for a new high-altitude Antarctic base. At near-IR wavelengths away from $\mathrm{OH}$ airglow lines (such as the long wavelength portion of the $\mathrm{K}$ window at $2 \mu \mathrm{m}$ ), the low temperature of the environment results in a sky that is more than 100 times darker during the Antarctic night than the night sky at temperate latitudes, allowing very sensitive near IR observations. Experiments operated during the Antarctic summer have confirmed expectations about the mm and sub-mm transparency of the sky during the 'warm' months. A $1 \mathrm{~mm}$ wavelength heterodyne 'tipper' is being installed to monitor the mm-wavelength sky emission through the 1992 Austral winter. By the following winter, we hope to install a near infrared sky brightness sensor to directly measure the night sky flux in the $2 \mu \mathrm{m}$ window and an optical seeing monitor to measure stellar image motion and diameters to evaluate the impact of the strong thermal inversion over the Polar ice. These instruments will also determine the impact of Polar Stratospheric Clouds and cirrus on near-infrared observations.

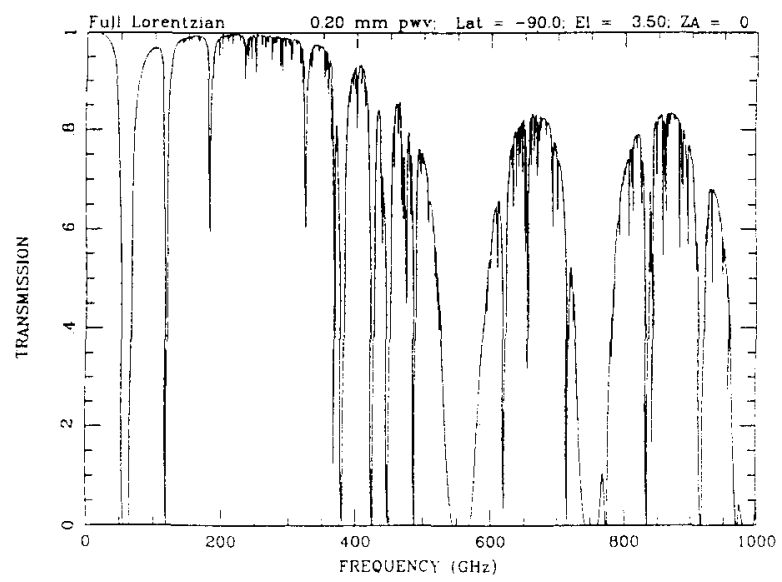

Figure 1. Model atmospheric transmission computed for the pressure altitude of the South Pole $(3500 \mathrm{~m})$ and $0.2 \mathrm{~mm}$ precipitable water vapour. 